\section{Ribonuclease still under attack}

\author{
from Barry Robson
}

THE enzyme ribonuclease has always been popular with experimentalists seeking to understand the relations between chemical formulae, three-dimensional structures, and functions of protein molecules. Ribonuclease is a particularly attractive weak spot in nature's defences because it was the first enzyme to have its chemical formula determined (as a sequence of amino acid residues), one of the earliest enzymes to have its three-dimensional structure elucidated, and the first protein for which it was carefully demonstrated that the chemical formula alone determines the three-dimensional structure and function. It continues to be a prime target in a number of recent investigations.

One important approach to understanding the relationship between the chemical formula and the final structure is to attempt to follow how a protein molecule folds up after it has been unfolded. In particular, it would be of interest to know how a protein folds up so rapidly: the current theory is that it is rapidly directed towards the native structure by a process of nucleation in which certain structural features such as $\alpha$-helices and $\beta$-pleated sheets form first, propagating further structure around them. By tritiating the exchangeable amide protons of unfolded RNase $A$ and following exchange of tritium with the surrounding solvent during refolding, Schmid and Baldwin (J. molec. Biol. 135, $199 ; 1979)$ have been able to confirm that the protein does fold up by a stepwise process, early stages being hydrogen bonded structures in which the tritium is protected from exchange. Since $\alpha$-helices and $\beta$-pleated sheets are such structures, this would seem at first consideration to support the nucleation model. In particular, it supports the idea that such intermediate structures are metastable, possibly in appropriate conditions stable, and form relatively rapidly, as suggested previously by studies on penicillinase using more standard techniques (Robson \& Pain Biochem. J. 155, 325; 1976).

However, the nucleation idea is complicated by the experimental observations that the stability of the intermediate structure depends on interactions with the rest of the protein. In their case Schmid and Baldwin find that an artificially cleaved-off fragment of ribonuclease known as the S-peptide seems to be essential for the stability of the intermediates. In a further detailed study

Barry Robson is in the Department of Biochemistry, University of Manchester.
Labhardt and Baldwin (J. molec. Biol. 135,231 and $245 ; 1979$ ) observed (1) that $S$ protein (ribonuclease A without S-peptide) folds up some hundred times more slowly than intact ribonuclease $A$, and (2) that on adding S-peptide to S-protein, the combination of S-peptide and S-protein occurred at an intermediate stage, rather than at the end of the folding process. Labhardt and Baldwin exclude the model for protein folding in which there is an initial slow diffusion of the molecular conformation into a restricted range of three-dimensional structures, and demonstrate instead successive folding stages with rates dependent on the stabilities of intermediates from preceding steps. Whether this supports or excludes the classical model for nucleation depends on how one interprets that model. Rightly or wrongly, many workers seem to associate the idea of nucleation with a key directing step, the question of rates being one of their own preference. That Labhardt and Baldwin are more proper about the definition of nucleation is, however, irrelevant, since they provide a self-consistent and meaningful description of the folding process.

Baldwin and colleagues also give considerable attention to the fast and slow folding species of RNase in their studies. In this they accept the current interpretation that the slower species is the product of a kind of isomerisation of proline residues in the unfolded form. This may, however, be a case in which attention to one enzyme is less satisfactory. The interpretation in terms of isomerisation is best tested by reference to the different rates of folding kinetics of many different proteins, correlating the proline content with those rates (Stellwagen J. molec. Biol. 135, 217; 1979).

Studies of this type provide a logical framework for the theoreticians, whose principal goal is to predict the threedimensional structure of a protein from its amino acid sequence alone, and ultimately to design new proteins by chosing the amino acid sequence to give the required conformation and function. A major difficulty here is that many amino acid residues considered alone have only a subtle effect on the overall conformation of the molecule: all the amino acid residues tend to interact in a complex way. The theoretician is faced with having to handle calculations concerning a delicate balance of forces and it is doubtful whether his armoury is as yet adequate to this task. Gutte, Daumingen and Wittscheiber (Nature 281, 650; 1979) recently mounted an audacious rear attack on this problem by designing an artificial RNase of 34 residues, having chosen amino acid residues and their sequence which would seem likely to be a very strong generator of the conformation required. Particular emphasis was given to the strong tendencies of certain residues to form $\alpha$-helix or $\beta$-pleated sheet. The philosophy was that the more subtle and ambiguous aspects should be avoided, and this rationale seems justified by their subsequent synthesis and investigation of the protein. Briefly, although a detailed analysis of the three-dimensional structure was not possible, the protein indeed had significant activity, particularly when in a dimerised form.

More precisely, one should say that the production of RNase activity may have been something of a bonus, since they state that their immediate aim was to produce a protein with nucleic acid binding ability. This is a necessary but not sufficient condition for RNase activity and it is conceivable that catalytic activity might have to have been introduced at a later stage by further refinement of the sequence. This turned out not to be the case, and it seems that their work brings the status of the theoretician's art one step nearer to practical application. However, noting that the activity of the artificial RNase was only a few per cent of natural RNase, one should consider appropriate control experiments. As Gutte et al. discussed, random amino acid sequences often have significant enzymic activities of various types. Perhaps it would have been helpful to compare the activity of their artificial RNase with that of random amino acid sequences of similar length and composition to establish the importance of specific consequences. If the histidine residue was responsible for the activity, then the precise environment may have been rather less important. Further, catalytic activity of another kind has been associated with a mere 10-residue sequence (Chakravarty et al. Experientia 29, 786; 1973), so it does not follow that all of the artificial molecule was in the expected conformation. Particularly when the substrate is a macromolecule, this may itself be a source of the appropriate environment and conformational stability, involving a kind of 'induced fit' of the synthetic protein.

RNase and their relatives are among some of the smallest enzymes known and their ability to interact with nucleic acid makes them useful models both for modern and prebiotic systems of more general interest. Undoubtedly the recent studies will encourage the continued use of RNase and its synthetic analogues.

\section{Correction}

In the article 'Probing the insulin receptor' (News \& Views 282, 11; 79) the incorrect impression may have inadvertently been given that the photolabelled probes used by C.C. Yip et al. were less well characterised than those of other groups. (line 4, paragraph 3, page 11). The probes are in fact specifically photolabelled at the B-29, A1-B29 or B1 positions, which was not stated in the article. 